BMJ Open Sport \& Exercise Medicine

\section{Empowering the health of retired professional footballers: the systematic development of an After Career Consultation and its feasibility}

To cite: Gouttebarge V, Goedhart E, Kerkhoffs G. Empowering the health of retired professional footballers: the systematic development of an After Career Consultation and its feasibility. BMJ Open Sport \& Exercise Medicine 2018;4:e000466. doi:10.1136/ bmjsem-2018-000466

Accepted 16 November 2018

Check for updates

(C) Author(s) (or their employer(s)) 2018. Re-use permitted under CC BY-NC. No commercial re-use. See rights and permissions. Published by BMJ.

'World Players' Union (FIFPro), Hoofddorp, The Netherlands

${ }^{2}$ Amsterdam UMC, Univ of Amsterdam, Department of Orthopaedic Surgery, Amsterdam Movement Sciences, Meibergdreef 9, Amsterdam, The Netherlands

${ }^{3}$ Academic Center for Evidencebased Sports Medicine (ACES), Academic Medical Center, Amsterdam, The Netherlands ${ }^{4}$ Amsterdam Collaboration for Health \& Safety in Sports (ACHSS), AMC/NUmc IOC Research Center, Amsterdam, The Netherlands

${ }^{5}$ Division of Exercise Science and Sports Medicine, University of Cape Town, Cape Town, South Africa

${ }^{6}$ Royal Netherlands Football Association (KNVB), FIFA Medica Center of Excellence, Zeist, The Netherlands

Correspondence to Dr Vincent Gouttebarge; $v$ gouttebarge@amc.uva.nl

\section{ABSTRACT}

Objectives This article describes (1) the systematic development of the intervention and (2) the assessment of its feasibility (in terms of relevancy, suitability, satisfaction and added value).

Methods The intervention was developed based on the first four steps of the Intervention Mapping and Knowledge Transfer Scheme processes. Subsequently, a qualitative research based on a one-group post-test crosssectional design was conducted. Eight retired professional footballers underwent the developed intervention and its feasibility (operationalised in relevancy, suitability, satisfaction and added value) was assessed by means of a questionnaire and interview.

Results An After Career Consultation (ACC) was developed with a focus on five main domains: (1) detraining from professional football; (2) remission of osteoarthritis; (3) promotion of healthy lifestyle; (4) preventing mental and cognitive health problems; and (5) employment and education. The ACC relies on three components: (1) raising self-awareness about potential physical, mental and social health problems after a career in professional football; (2) medical examination (60 $\mathrm{min}$ ) with thorough medical history and general physical examination; and (3) follow-up during 3 months (if necessary) about optimal skills and strategies to empower physical, mental and social health and quality of life. The relevancy, suitability, satisfaction and added value of the ACC were positively evaluated by the retired professional footballers.

Conclusion The ACC was developed with a focus on five main health-related domains. The feasibility of the ACC was positively assessed by retired professional footballers, while the suggestion was made to repeat the ACC in the initial years after football retirement.

\section{INTRODUCTION}

Transitioning out of professional, that is, elite, sport is not easy. During this transitioning process, retired athletes might face several challenges such as adjusting to a new life and lifestyle, being suddenly 'like everyone else' or missing the atmosphere of sport and competition, but especially dealing with both physical and mental health problems. ${ }^{1}$ The scientific

\section{What are the new findings?}

- Based on the Intervention Mapping and Knowledge Transfer Scheme processes, the After Career Consultation was developed in order to empower the sustainable physical, mental and social health, and the quality of life of retired professional footballers.

- The After Career Consultation focuses on five main domains: (1) detraining from professional football; (2) remission of osteoarthritis; (3) promotion of healthy lifestyle; (4) preventing mental and cognitive health problems; and (5) employment and education.

- The relevancy, suitability, satisfaction and added value of the After Career Consultation were positively evaluated by the retired professional footballers.

literature has shown that osteoarthritis (OA) in the lower limbs is highly prevalent among relatively young (45 years old or younger) retired professional footballers, ranging from $17 \%$ for ankle OA to $80 \%$ for knee OA. ${ }^{2}{ }^{3} \mathrm{~A}$ recent study confirmed these findings and found a prevalence of lower extremity OA of 33\% among 396 retired professional footballers (mean age 36 years). ${ }^{4}$ The adverse impacts of this joint disease on the quality of life and functioning (work and daily living) cannot be ignored: (1) retired professional footballers suffering from OA have a significant lower level of health-related quality of life than those without OA; the large majority $(90 \%)$ of retired professional footballers suffering from OA report moderate or severe joint pain and discomfort; (2) around $65 \%$ indicates experiencing moderate or severe problems with mobility or performing everyday activities (work, study, household). ${ }^{45}$ Also, mental health problems were found to be common among retired professional footballers, with a prevalence rate ranging from $24 \%$ for adverse alcohol use to $29 \%$ for sleep disturbance. ${ }^{6}$ Two recent studies among former elite athletes (including footballers) have suggested that these mental health problems 
might be associated with sport career-related concussions and with postsport OA. ${ }^{78}$

Despite the physical and mental health problems that might occur when transitioning out of football, both current and retired professional footballers have repeatedly mentioned that postfootball support measures were not yet available. ${ }^{9}{ }^{10}$ In 2014, the World Players' Union (FIFPro) introduced the concept of an exit-career examination as a potential intervention for recently retired professional footballers. ${ }^{11}$ Subsequently, a scientific research project led by FIFPro, in collaboration with the Academic Medical Centre Amsterdam (the Netherlands) and the Royal Dutch Football Association, initiated plans to develop and implement an intervention to empower the physical, mental and social health of retired professional footballers. This article describes: (1) the systematic development of the intervention; and (2) the assessment of its feasibility (in terms of relevancy, suitability, satisfaction and added value) among retired professional footballers.

\section{SYSTEMATIC DEVELOPMENT OF THE INTERVENTION}

In health promotion and sports research, interventions have been developed following two similar structured and systematic processes: Intervention Mapping (IM) and the Knowledge Transfer Scheme (KTS). ${ }^{12-14}$ Both approaches rely on several sequential steps that lead to the development, implementation and evaluation of an intervention, namely: (1) devising a needs assessment; (2) formulating the objective and target group of the intervention; (3) selecting contents for the intervention; (4) developing the intervention; (5) developing an adoption and implementation plan; and (6) developing an evaluation plan. ${ }^{12-14}$ Accordingly, the first four steps of the IM and KTS structured and systematic processes were applied in order to develop an intervention aiming to empower the physical, mental and social health of retired professional footballers.

\section{Needs assessment (step 1)}

The key purposes of the needs assessment were: (1) to assess the needs for a postsport intervention; (2) to identify the objectives and target groups of the intervention; and (3) to discuss strategies for the future implementation of the intervention. To this end, interviews were held with several key stakeholders within professional football, namely eight current professional footballers, eight retired professional footballers, eight representatives of footballers' unions and eight club physicians. All participants were approached by the researchers and gave their informed consent to participate. Open questions were formulated in order to gather a broad perspective of perceptions and opinions of the participants, especially on: (1) the current support given to retired professional footballers; (2) the needs for a postsport intervention; and (3) the strategies to deliver the intervention. Before each interview (lasting around $15 \mathrm{~min}$ ), participants received standard information about its purpose, duration and procedures. The interviews were led by an experienced interviewer who took a facilitator role, asking actively for further explanations of answers given when necessary. The interviews were audiotaped, and notes were taken. All interviews were conducted principally between football seasons in 2014, 2015 and 2016. Subsequently, an electronic questionnaire (in Dutch, English, French and Spanish) was set up and distributed to current and retired professional footballers. Information gathered previously through the interviews was partly used to formulate questions related to: (1) the current support after a career in professional football; and (2) the needs towards a postsport intervention. In order to guarantee the strict confidentiality of the responses, no personal identifiable information was included in the questionnaire. Information about the questionnaire was sent by email to potential participants by FIFPro. If wanting to participate, participants gave their informed consent and were asked to anonymously complete their electronic questionnaire within 2 weeks. One reminder was sent per email after 2 weeks. Once completed (around $10 \mathrm{~min}$ was needed), the electronic questionnaires were saved automatically on a secured electronic server. The questionnaire was distributed online between August 2015 and February 2016.

Information collected through the interviews indicated that: (1) the long-term physical, mental and social health of professional footballers was being neglected; (2) no support for players after their professional football career was available; (3) psychosocial support and care was needed; (4) players should receive counselling on lifestyle; (5) players should receive advice for detraining after their career; and (6) support and care for retired professional footballers should be centrally organised. Some results of the interviews have been published elsewhere, ${ }^{8}$ while three examples of quotes retrieved from these interviews can be found in Box 1. This qualitative information was confirmed by the outcomes of the questionnaire completed by 194 retired professional footballers. The participants had a mean age of 35 years, a professional football career with a mean duration of 12 years, and the mean duration of their retirement was 4 years. Some results of the questionnaire have been published elsewhere, ${ }^{10}$ while the main outcomes of the questionnaire are presented in Box 1.

\section{Objective(s) and target group(s) of the intervention (step 2)}

First, three systematic literature studies related to OA were performed, while two observational epidemiological studies about the physical, mental and social health of retired professional footballers were conducted. ${ }^{2-8}$ Second, an electronic questionnaire (in Dutch, English, French and Spanish) was set up and distributed to 70 representatives of footballers' unions from all continents (December 2015). Information gathered previously through the scientific literature was partly used to formulate questions related to the content of a postsport intervention and its future implementation. If wishing 
Box 1 Needs assessment towards an intervention after a professional football career

Quotes from interviews:

- 'Players who are nearing the end of their career must seek support from people who can help them prepare for what is going to happen, for instance representatives from the players' association or physicians.'

- 'I didn't have any medical care and support after my career. I would have liked to have had some because of the physical and mental changes I experienced.

- 'All stakeholders within professional football have the responsibility to offer proper medical care and support to professional footballers after their career, directed towards either their physical or psychosocial health.'

Main outcomes of the questionnaire:

- Nearly one out of two respondents stated that their health substantially influenced their quality of life.

- Eighty-one per cent of the respondents mentioned that there was a lack of sufficient support directed towards health problems after a career in professional football.

to participate, participants gave their informed consent and were asked to anonymously and instantly complete the questionnaire (ParticiPoll, Leicester, UK). Lastly, all information collected was discussed during a meeting by four experts in the field of professional football and football medicine in order to clearly formulate the objective(s) and target group(s) for the intervention.

Based on the scientific literature and empirical evidence collected, three main health areas were identified as relevant for retired professional footballers, namely: (1) OA: retired professional footballers are likely to suffer from OA (prevalence ranging from $17 \%$ for ankle OA to $80 \%$ for knee $\mathrm{OA}$ ) and $\mathrm{OA}$ is likely to impair functioning and quality of life; (2) symptoms of common mental disorders $(\mathrm{CMD})$ : prevalence among retired professional footballers ranges from $18 \%$ for symptoms of distress to $35 \%$ for symptoms of anxiety/depression; (3) adverse lifestyle: prevalence among retired professional footballers reaches nearly $25 \%$ for adverse alcohol use and $65 \%$ for adverse nutritional habits. In addition, it was shown that being employed and having a higher number of working hours was favourable for the mental health of retired professional footballers. ${ }^{15}$ Furthermore, the needs assessment revealed that detraining was a relevant postsport aspect to take into account within the intervention. The electronic survey completed by 59 representatives of footballers' unions ( $84 \%$ response rate) confirmed the previous findings. Furthermore, $97 \%$ of the respondents mentioned that an intervention should be offered as a consultation at the end of the players' career.

Based on the aforementioned, experts formulated the following intervention objective, namely to empower the sustainable physical, mental and social health, and the quality of life of retired professional footballers with a focus on detraining, OA, symptoms of CMD, adverse lifestyle and employment/education.

\section{Development of the intervention (steps 3 and 4)}

In order to develop the intervention, all information gathered in steps 1 and step 2 was synthetised, analysed, discussed and translated during three meetings (2 hours each) with four experts in the field of professional football and football medicine (April to September 2016). The intervention (available fully in Dutch) consists of an After Career Consultation (ACC) aiming to empower the sustainable physical, mental and social health, and quality of life of retired professional footballers. The ACC focuses on the following five main domains: (1) detraining from professional football; (2) remission of OA; (3) promotion of a healthy lifestyle; (4) preventing mental and cognitive health problems; and (5) employment and education. In order to cover these five domains, the ACC relies on the following three components:

1. Raising self-awareness of the retired professional footballers about the five main domains of the ACC.

2. Medical examination of $60 \mathrm{~min}$ in order to identify and discuss potential health problems and advise players about these problems (if any).

3. Follow-up during 3 months (if necessary) of retired players about optimal skills and strategies to empower their physical, mental and social health and quality of life.

Prior to the medical examination, retired professional footballers receive information about potential challenges after a professional football career and about the ACC. Raising self-awareness of the retired professional footballers about these challenges is important in order to optimally prepare the medical examination. Figure 1 presents the informative leaflets (in Dutch) sent to retired players. Retired professional footballers are also requested to complete a comprehensive general examination questionnaire, information that is used during the medical examination.

The medical examination is delivered centrally according to a standardised protocol (Box 2) by a sports physician with extended expertise in professional football. The medical examination starts with a thorough medical history (including the validated 12-item General Health Questionnaire for psychological symptoms) and a general physical examination related to all major body systems (especially respiratory, cardiovascular, nervous and musculoskeletal systems). ${ }^{16} 17$ Based on the information retrieved, potential health problems are further discussed and advice for the upcoming period is given to the retired professional footballer. Depending on the findings and advice given during the medical examination, retired players are followed (face-to-face and/or by phone) during a period of 3 months in order to discuss the given advice, monitor their advancements and explore their potential further needs.

\section{FEASIBILITY OF THE INTERVENTION Methods}

A qualitative research based on a one-group posttest cross-sectional design was conducted by means of 


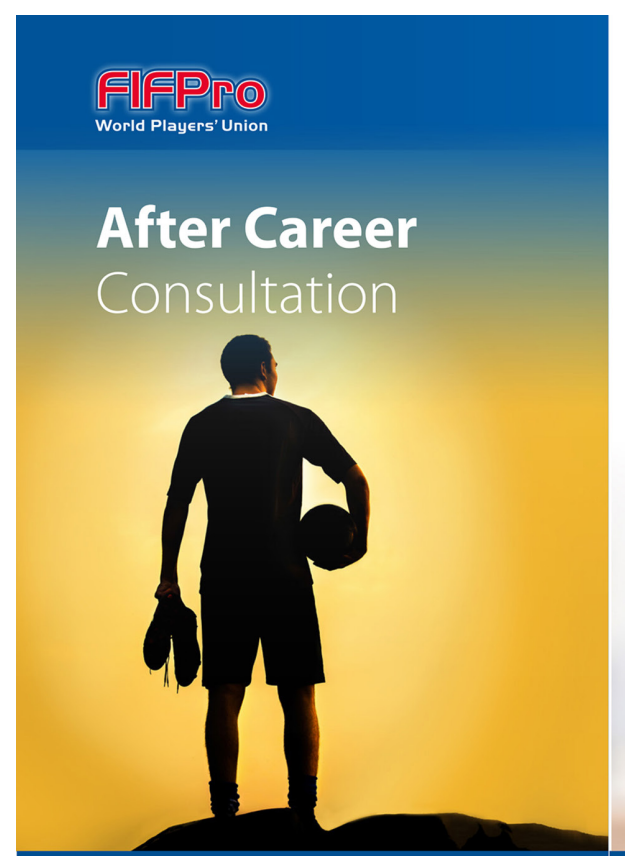

\section{Introduction}

After finishing a football career, a new stage in life begins for each player. This moment often coincides with questions about the player. This moment often coincides with questions about the
physical and mental health of the former footballer: how can I stay physical and mental health of the former footballer: how can I stay pain, and am I mentally prepared for retirement?

FIFPro developed the After Career Consultation to empower th physical, mental and social health and quality of life of retire professional footballers. The After Career Consultation gives players the possibility to receive advice for their future life, focussing on four principal domains: Detraining, Healthy Lifestyle, Joint Pain and Mental Health. This leaflet presents basic information about these four domains.

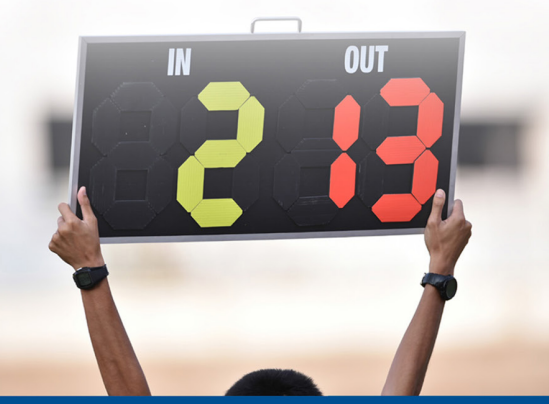

Changes after retirem
professional football After retiring from professional football, a player can experience important changes. Potential physical changes: tion of stamina and aerobic capacity; - Reduction or - Weight gain; Possible mental changes: Possible mental changes:
- Change in daily schedule and daily routine - Reduction of (public) attention; - Lack of goals for the future, - Withdrawal symptoms:

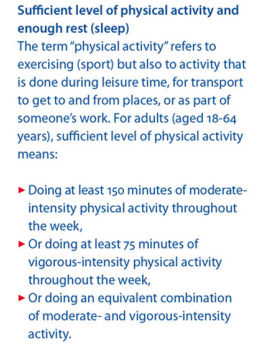
activity.

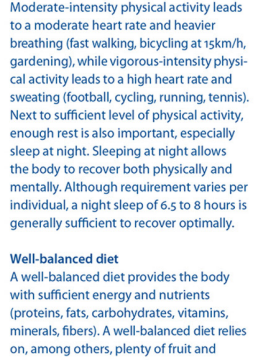

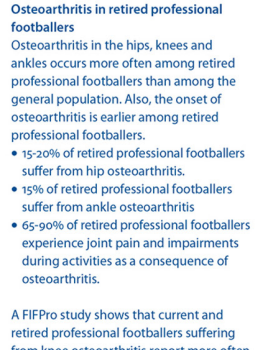

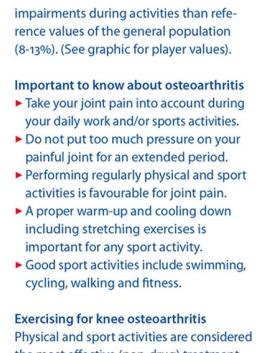

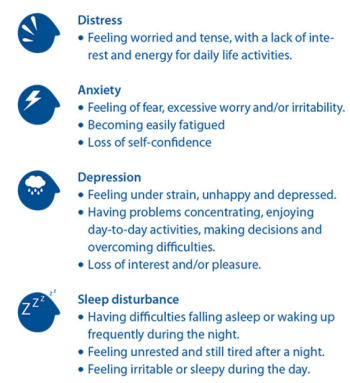

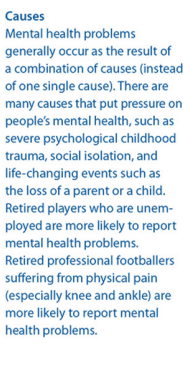

Figure 1 After Career Consultation: information for retired professional footballers.

questionnaires and semistructured interviews. ${ }^{18}$ This study was done in accordance with the Declaration of Helsinki, while ethical approval for the study was provided by the Medical Ethics Review Committee of the Academic Medical Center (W16_190\#16.223; Amsterdam, the Netherlands). ${ }^{19}$

The participants were retired professional footballers. Inclusion criteria were: (1) between 30 and 40 years of age; (2) male; (3) member of the Dutch national footballers' union (Vereniging van Contractspelers); (4) having played professional football in the highest or second highest league for at least five seasons; (5) retired from professional football within the past 12 months; and (6) speaking and reading Dutch fluently. Because of the qualitative nature of the study, no sample size calculation was conducted, striving to invite the Dutch footballers who retired from professional football after the season 2015-2016 and who fulfilled our inclusion criteria.

Feasibility was operationalised by measuring the relevancy, suitability, satisfaction and added value of the ACC, using both questionnaires and semistructured interviews. Single statements related to relevancy and added value were formulated (eg, 'The After Career Consultation has added value for players once they retire from professional football') for each domain of the ACC (10 statements related to the domains and two related to the ACC as a whole) and measured on a 5-point scale (from 'totally disagree' to 'totally agree'). The semistructured interview was related to the suitability of the ACC and the 
Box 2 After Career Consultation: overview of the medical protocol

\section{Characteristics}

Age, length, body weight, education, marital situation, employment status, lifestyle.

\section{Football history}

Playing position, duration of football career, number of matches played last season, current training and competition load, preferred kicking leg.

\section{Medical history}

Musculoskeletal injuries, surgeries, concussions, family history, medication use, lifestyle, allergies, respiratory system, cardiovascular system, current complaints.

\section{Mental health}

This 12-item General Health Questionnaire (GHQ-12) validated in several languages (including Dutch, English, French and Spanish) assesses minor psychiatric disorders (especially symptoms of anxiety/depression) in the previous weeks (eg, 'Have you recently felt under strain?'). ${ }^{1516}$ Based on the traditional scoring system (' 0 ' for a favourable answer; ' 1 ' for an unfavourable answer), a total score ranging from 0 to 12 is calculated by summing up the answers on the 12 items, with a score of 3 or more indicating the presence of minor psychiatric disorders. ${ }^{1516}$

\section{Physical examination}

- Height, weight, body fat percentage, vision, blood pressure.

- General routine screening internal diseases, heart with ECG at rest, lungs (pulmonary function), nerve system neurology and ear-nose-throat.

- Examination of the musculoskeletal system: range of motion joints, muscle function and flexibility, signs of overuse tendon insertions, active and passive stability.

- Stress testing on bicycle ergometer with continuous ECG monitoring. Additional examinations

Musculoskeletal ultrasound, neuropsychological tests, lab analysis on indication and if desired.

\section{Current and future activities}

Daily life, sport and exercise, education and employment.

\section{Advices}

Based on the outcomes, the retired player is being informed on the conditions. The advices on how to deal with the results are individualised in accordance with the personal situation and desires of the retired player.

satisfaction of the participants, including in total eight questions.

An electronic questionnaire was set up in Dutch, including all statements related to the feasibility of the ACC. In addition, the following descriptive variables were added: age, height, weight, duration of football career, number and nature of severe injuries (and surgeries) during football career, duration and nature of retirement, and employment status. Participants were informed by the Dutch national footballers' union (Vereniging van Contractspelers) and FIFPro about the ACC and related feasibility study. If willing to be enrolled, participants gave their informed consent and underwent the ACC. Within 4 weeks of completing the ACC, participants filled in the electronic questionnaire (5-10 min needed). Three months after the ACC, all interviews were conducted by telephone (10-15 min needed), with written notes being made by the interviewer (VG). All responses collected through questionnaires and interviews were anonymised for privacy and confidentiality reasons, and were saved automatically on a secured electronic server that only the principal researcher could access. Retired professional footballers participated voluntarily in the study and did not receive any reward for their participation. Based on the responses from all participants from the questionnaires and interviews, a content analysis was conducted (coding, categorising and summarising the data) for each participant in order to present a valid and thorough picture of their views and thoughts on the feasibility of the ACG.

\section{Results}

Of the 23 footballers fulfilling our inclusion criteria, eight participants gave their written informed consent to participate in the feasibility study and to undergo the ACC. The mean age, height and weight of the participants were 34 years $(\mathrm{SD}=1), 185 \mathrm{~cm}(\mathrm{SD}=5)$ and 82 $\mathrm{kg}(\mathrm{SD}=7)$, respectively. On average, participants had competed in professional football for 13 years $(\mathrm{SD}=4)$ and had suffered from four time-loss (14 days or more) injuries during their career. One participant had suffered from one concussion, one from two concussions and one from eight concussions (recovery within 2 weeks for all of them). Only one participant had been forced to retire from football. All but one were employed at the time of the study. The ACC revealed the following medical conditions:

- Heart rhythm disturbance $(\mathrm{n}=1)$.

- Abnormal stress-ECGs $(\mathrm{n}=2)$.

- Knee complaints with limited range of motion (ROM) and signs of arthrosis $(\mathrm{n}=1)$.

- Elbow complaints with limited ROM $(\mathrm{n}=1)$.

- Limited hip function without complaints $(\mathrm{n}=2)$.

- Patellar tendinopathy $(\mathrm{n}=1)$.

- Achilles tendinopathy with complaints confirmed by ultrasound $(n=1)$.

With regard to the following, advices were given: (adapted) physical exercise and training $(n=3)$; referral for further examination $(n=2)$; altered nutrition $(n=2)$; use or adapt insoles $(n=2)$; and referral for manipulative medicine $(\mathrm{n}=1)$.

The outcomes of the electronic questionnaire revealed that all participants were highly positive with regard to the feasibility of the ACC. All five main domains of the ACC were assessed as relevant or highly relevant. The ACC as a whole received a positive appraisal, with high degree of relevancy and added value reported by the participants. These findings were confirmed by the players' narratives, mentioning that 'All five main domains of the ACC are of added value, especially education and employment' and that 'leaflets including relevant information could be of added value for most players and should be given in the early years of a football career.' With regard to the future implementation of the ACC, participants mentioned 
that players' unions were the key actors for the support of retired professional footballers but emphasised also that the collaboration of these players' unions with the national football associations would be essential in order to implement sustainably the ACC. Concerning the potential role of the clubs, participants stated that "clubs were focusing on the short term and consequently were not likely to invest in the long-term well-being of players.' A summary of an interview held with a retired professional footballer is presented in Box 3.

\section{DISCUSSION}

This article aimed to describe: (1) the systematic development of an intervention to empower the physical, mental and social health of retired professional footballers; and (2) the assessment of its feasibility (in terms of relevancy, suitability, satisfaction and added value). The systematic development of the intervention was based on two structured and systematic processes, involving experts in professional football and sports medicine. As a result, the ACC was developed with a focus on five main domains: (1) detraining from professional football; (2) remission of OA; (3) promotion of a healthy lifestyle; (4) preventing mental and cognitive health problems; and (5) employment and education. The feasibility of the ACC in terms of relevancy, suitability, satisfaction and added value was positively evaluated by the retired professional footballers.

\section{Unique support for retired professional footballers}

During their career, professional footballers are extensively screened and investigated for health-related problems, especially musculoskeletal injuries. These repeated medical assessments are well documented and even made mandatory by the football governing bodies. By contrast, after their football career, professional players do not have access to suitable and formal medical support. ${ }^{20}$ This is striking in view of the fact that retired professional footballers have an increased risk of physical, mental and social health problems. Consequently, the developed ACC can be seen as a unique measure offering retired professional footballers guidance and support on diverse relevant health-related domains. Most importantly, both current and retired professional footballers have already expressed their needs for this level of medical care and support. ${ }^{9} 10$

\section{Alterations to the ACC}

As a consequence of the feedback from the retired professional footballers during our feasibility study, the main alteration to the ACC that should be considered before its future implementation is related to its frequency. For our feasibility study, we chose to offer the ACC and its related 3-month follow-up (face-to-face and/ or by phone) to retired professional footballers just once. During the conducted evaluation, most of the participants mentioned that the ACC could also be repeated several times in the initial years of retirement as the
Box 3 View of a retired professional footballer on the After Career Consultation

Being 33 years old at the time of the After Career Consultation (ACC), this retired professional footballer had played around 250 official professional football matches over 15 seasons. During his career, he suffered from several severe musculoskeletal injuries: (1) rupture of the ACL in the left knee (age: 20 years) that was surgically treated; (2) rupture of the ACL in the right knee (age: 23 years) that was surgically treated; and (3) deep vein thrombosis in the left leg. This player mentioned that after these severe injuries, he never regained his initial level of play and thus never reached his full potential. After the 20152016 season, he retired voluntarily from professional football and he is now employed as a youth football trainer. At the time of the ACC, he did not experience any mental health problems but he was still experiencing some thrombosis-related pain in his leg (no impairments during daily life, sport or work activities).

Interviewer. Did you, during or after your career, receive any information from your club or union about the transitioning period? Retired player: No, I was never informed about the problems that might occur after retirement from professional football.

Interviewer. Would you have liked to receive information about the period of transition out of professional football at an early stage? Retired player. Yes, I would have liked to receive this information a few years ago. For instance, an extensive booklet focusing on all relevant post-football aspects could be developed and distributed by players' unions to all players. Perhaps to all players once they are 25 years old This information and related general advice is particularly relevant for those that are forced to retire. Also, this booklet might play a positive role in the preparation of the after-football period and might stimulate players to think about their future post-football plan.

Interviewer. We developed the After Career Consultation for the needs of professional footballers. Was the After Career Consolation of added value for you?

Retired player. Yes, it was of added value for me. Perhaps not right now because I feel good generally at the present time but it has warned me about potential problems that might arise in the future. Interviewer. Are all five domains relevant within the After Career Consolation? Do you have another domain to suggest?

Retired player. Yes, I believe that the consult covers all relevant domains, but I guess that colleagues might have other needs. This should be explored during the consultation. I guess also that some relevant information could be given to the partner of the retired professional footballer.

Interviewer. What is your view on the form of the After Career Consultation (duration, form, domains, location, collaboration...)? Retired player. The location, duration, form and follow-up of the After Career Consultation was good. The only remark I have is that the After Career Consultation could be offered to retired players not only once but perhaps after two or three years, depending on the needs of course.

Interviewer. We want to implement the After Career Consultation in the next few years. Any ideas about how we can do that?

Retired player. A good collaboration between unions, clubs and federations would be ideal of course. The collaboration between a players' union and a football association is essential in order to implement sustainably the After Career Consultation. It would be nice and fair if clubs were interested and supportive, but l'm not very hopeful that clubs will be. 
physical and mental state of players might evolve during this period. Consequently, the ACC should be offered to retired professional footballers at regular intervals in the initial years after retiring from football, depending on their medical status and needs. This should be especially relevant for those players forced to retire or those without further employment plans. ${ }^{1521}$ Such a recurring ACC is likely to optimally empower the sustainable physical, mental and social health of retired professional footballers, leading to a successful transition out of sport and thus to a higher and sustainable quality of life.

\section{Challenges for future implementation}

With regard to its positive evaluation during our feasibility study, the ACC should be implemented in professional football in order to empower the sustainable physical, mental and social health and quality of life of retired professional footballers. A main lesson learnt from our feasibility study is that such an implementation should be discussed with, and facilitated by, all stakeholders involved in professional football. Especially the national footballers' unions and football associations should be collaborating to offer the medical support needed by players during their transition out of football. Ideally, clubs should also be involved in the management of retired professional footballers, for instance, by allowing the use of their medical staff to conduct an ACC.

Financing the ACC and its potential subsequent treatments in a sustainable way will be a major challenge. However, the authors would like to emphasise that in 2009, the WHO and International Labour Organization stated, 'the protection, promotion, surveillance and maintenance of the highest degree of physical, mental and social well-being of workers in all occupations long after they enter their retirement years is not only a labour right but a fundamental human right that should be facilitated by social partners and stakeholders. ${ }^{22}$ Consequently, it seems logical that the ACC should be financed by all stakeholders within professional football, namely clubs (employers), players' unions (employees) and football associations (governing bodies). This collaboration should enable the proper management of retired professional footballers, empowering their sustainable physical, mental and social health and quality of life.

\section{CONCLUSION}

The ACC was developed with a focus on five main domains: (1) detraining from professional football; (2) remission of OA; (3) promotion of a healthy lifestyle; (4) preventing mental and cognitive health problems; and (5) employment and education. The feasibility of the ACC was positively assessed by retired professional footballers, while the suggestion was made to repeat the ACC in the initial years after football retirement. The ACC should be implemented in professional football, facilitated ideally by all stakeholders including clubs (employers), players' unions (employees) and football associations (governing bodies).
Acknowledgements We greatly thank all experts (current and retired players, representatives of footballers' unions, club physicians) involved in the development of the intervention. We are also thankful to the retired professional footballers for their participation in the feasibility study.

Contributors VG and GK were responsible for the conceptualisation of the ACC. All authors were responsible for the final development of the ACC, as well as for the definition, preparation and execution of the study. VG was responsible for the analyses and the preparation of the manuscript. EG and GK were responsible for the critical review of the manuscript. All authors read and approved the final manuscript.

Funding The authors have not declared a specific grant for this research from any funding agency in the public, commercial or not-for-profit sectors.

Competing interests None declared.

Patient consent for publication Not required.

Provenance and peer review Not commissioned; externally peer reviewed.

Open access This is an open access article distributed in accordance with the Creative Commons Attribution Non Commercial (CC BY-NC 4.0) license, which permits others to distribute, remix, adapt, build upon this work non-commercially, and license their derivative works on different terms, provided the original work is properly cited, appropriate credit is given, any changes made indicated, and the use is non-commercial. See: http://creativecommons.org/licenses/by-nc/4.0/

\section{REFERENCES}

1. Wylleman P, Reints A, De Knop P. A developmental and holistic perspective on athletic career development. In: Sotiaradou P, Bosscher D V, eds. Managing high performance sport. New York: Routledge, 2013.

2. Kuijt MT, Inklaar H, Gouttebarge V, et al. Knee and ankle osteoarthritis in former elite soccer players: a systematic review of the recent literature. J Sci Med Sport 2012;15:480-7.

3. Gouttebarge V, Inklaar H, Backx F, et al. Prevalence of osteoarthritis in former elite athletes: a systematic overview of the recent literature. Rheumatol Int 2015;35:405-18.

4. Gouttebarge V, Aoki H, Kerkhoffs G. Lower extremity osteoarthritis is associated with lower health-related quality of life among retired professional footballers. Phys Sportsmed 2018;46:471-6.

5. Gouttebarge V, Inklaar H, Frings-Dresen MHW. Osteoarthritis related consequences of a professional football career: a systematic review of the recent literature. J Sports Med Phys Fitness 2014;54:494-504.

6. Gouttebarge V, Aoki H, Kerkhoffs GM. Prevalence and determinants of symptoms related to mental disorders in retired male professional footballers. J Sports Med Phys Fitness 2016;56:648-54.

7. Gouttebarge V, Aoki H, Lambert M, et al. A history of concussions is associated with symptoms of common mental disorders in former male professional athletes across a range of sports. Phys Sportsmed 2017;45:443-9.

8. Schuring N, Aoki H, Gray J, et al. Osteoarthritis is associated with symptoms of common mental disorders among former elite athletes. Knee Surg Sports Traumatol Arthrosc 2017;25:3179-85.

9. Akturk A, Inklaar H, Gouttebarge V. Medical examinations in the Dutch professional football (soccer): a qualitative study. Int SportsMed J 2014;15:343-51.

10. van Ramele S, Aoki $\mathrm{H}$, Kerkhoffs GMMJ, et al. Mental health in retired professional football players: 12-month incidence, adverse life events and support. Psychol Sport Exerc 2017;28:85-90.

11. Gouttebarge V, Aoki H. Lifespan perspective of professional footballers' health. Asian J Sports Med 2014;5:e23813.

12. Bartholomew LK, Parcel GS, Kok G. Intervention mapping: a process for developing theory- and evidence-based health education programs. Health Educ Behav 1998;25:545-63.

13. Kok G, Gottlieb NH, Peters GJ, et al. A taxonomy of behaviour change methods: an Intervention Mapping approach. Health Psychol Rev 2016;10:297-312.

14. Verhagen $\mathrm{E}$, Voogt $\mathrm{N}$, Bruinsma $\mathrm{A}$, et al. A knowledge transfer scheme to bridge the gap between science and practice: an integration of existing research frameworks into a tool for practice. Br J Sports Med 2014;48:698-701.

15. Gouttebarge V, Aoki H, Verhagen E, et al. Are level of education and employment related to symptoms of common mental disorders in current and retired professional footballers? Asian J Sports Med 2016;7:e28447

16. Goldberg DP, Gater R, Sartorius N, et al. The validity of two versions of the GHQ in the WHO study of mental illness in general health care. Psychol Med 1997;27:191-7. 
17. Salama-Younes M, Montazeri A, Ismaïl A, et al. Factor structure and internal consistency of the 12-item General Health Questionnaire (GHQ-12) and the Subjective Vitality Scale (VS), and the relationship between them: a study from France. Health Qual Life Outcomes 2009;7:22.

18. Bowen DJ, Kreuter M, Spring B, et al. How we design feasibility studies. Am J Prev Med 2009;36:452-7.

19. World Medical Association. World Medical Association Declaration of Helsinki: ethical principles for medical research involving human subjects. JAMA 2013;310:2191-4.
20. Carmody S, Jones C, Malhotra A, et al. Put out to pasture: what is our duty of care to the retiring professional footballer? Promoting the concept of the 'exit health examination' (EHE). Br J Sports Med 2018. doi: 10.1136/bjsports-2017-098392. [Epub ahead of print 24 Mar 2018].

21. Brown JC, Kerkhoffs G, Lambert MI, et al. Forced Retirement from Professional Rugby Union is Associated with Symptoms of Distress. Int J Sports Med 2017;38:582-7.

22. International Labour Organization. Health and life at work: a basic human right. Geneva: International Labour organization, 2009. 